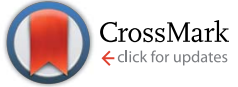

Cite this: RSC Adv., 2015, 5, 46430

\title{
Reversible magnetic mercury extraction from water
}

\author{
S. Fernandes, $\neq^{a}$ C. M. Eichenseer, $\neq^{a}$ P. Kreitmeier, ${ }^{a}$ J. Rewitzer, ${ }^{b}$ V. Zlateski, ${ }^{c}$ \\ R. N. Grass, ${ }^{c}$ W. J. Stark ${ }^{c}$ and O. Reiser ${ }^{\star a}$
}

\begin{abstract}
A facile and efficient way to decontaminate mercury(I) polluted water with the aid of magnetic, highly stable and recyclable carbon coated cobalt $(\mathrm{Co} / \mathrm{C})$ nanoparticles is reported. Comparing non-functionalised $\mathrm{Co} / \mathrm{C}$ nanomagnets with particles that were functionalised with amino moieties, the latter one proved to be more effective for scavenging mercury with respect to extraction capacity and recyclability. A novel nanoparticlepoly(ethyleneimine) hybrid (Co/C-PEI) prepared by direct ring opening polymerization of aziridine initiated by an amine functionalised nanoparticle surface led to a high capacity material $(10 \mathrm{mmol}$ amino groups per $\mathrm{g}$ nanomaterial) and thus proved to be the best material for scavenging toxic mercury at relevant concentrations ( $\mathrm{mg} \mathrm{L}^{-1} / \mu \mathrm{g} \mathrm{L}^{-1}$ ) for at least 6 consecutive cycles. On a large-scale, $20 \mathrm{~L}$ of drinking water with an initial $\mathrm{Hg}^{2+}$ concentration of $30 \mu \mathrm{g} \mathrm{L}^{-1}$ can be decontaminated to the level acceptable for drinking water $\left(\leq 2 \mu \mathrm{g} \mathrm{L}^{-1}\right.$ ) with just $60 \mathrm{mg}$ of Co/C-PEI particles.
\end{abstract}

Received 11th March 2015

Accepted 14th May 2015

DOI: $10.1039 / c 5 r a 04348 d$

www.rsc.org/advances

Facing the above-mentioned harms, different methodologies

\section{Introduction}

Removal of organic and inorganic waste from water has become an issue of major interest for the last few decades. In particular, the decontamination of toxic metals is still a matter of great concern, since these harmful substances can cause severe threats to human health. In this context, mercury is considered one of the most problematic pollutants to the environment and public health, being involved in several disasters of food poisoning in different countries around the world. ${ }^{1,2}$ The cumulative character of this metal leads to an enrichment in the environment and the food chain, ${ }^{3,4}$ which in turn may cause permanent adverse effects in the liver, lung, brain or kidney of living organisms, even at very low doses. ${ }^{\mathbf{1 , 4}}$ Furthermore, the solubility of mercury(II) ions in water brings along additional problems concerning the toxicity, especially for the aquatic system..$^{5}$ Indeed, its divalent form is often found in fresh water, seawater, ground water and soil in considerable amounts., Therefore, mercury and its derivatives are considered as priority hazardous substances (PHSs) ${ }^{\mathbf{1}, \mathbf{6}}$ by several environmental associations that have started mercury monitoring programs worldwide. $^{1}$

\footnotetext{
anstitute for Organic Chemistry, University of Regensburg, Universitätsstr. 31, 93053 Regensburg, Germany.E-mail: oliver.reiser@chemie.uni-regensburg.de

${ }^{b}$ Institute of Bioanalytical Chemistry, University of Regensburg, Germany

${ }^{c}$ Institute for Chemical and Bioengineering, ETH Zurich, Vladimir-Prelog-Weg 1, 8093 Zürich, Switzerland

$\dagger$ Electronic supplementary information (ESI) available. See DOI: 10.1039/c5ra04348d

\$ These authors contributed equally.
} have been used for water treatment such as centrifugation, ultrafiltration, crystallisation, sedimentation, solid-phase extraction and chemical precipitation. ${ }^{\mathbf{1 , 2}}$ Usually, the extraction of particular heavy metals is performed by using insoluble adsorbents. ${ }^{7}$ However, this method requires further filtration which involves energy-intensive pumping and tedious recovery of the materials. ${ }^{8}$

In an attempt to develop more sensitive, simple and costeffective materials, nanotechnology has attracted much attention in this field. ${ }^{2,5}$ Magnetic nanoparticles in particular might contribute to such applications due to their distinct advantages like high surface area-to-volume ratio and therefore higher extraction capacity compared to micrometer-sized particles. The most important benefits are the facile and convenient separation by applying an external magnetic field enabling an easy recovery and recycling of the scavenger, ${ }^{\mathbf{1 , 4 6}}$ potentially even in the open environment.

Additionally, materials that selectively bind $\mathrm{Hg}^{2+}$ in the presence of other metals are needed in order to prove feasibility in a real water decontamination situation. For instance, studies with 1-naphthylthiourea-methyl isobutyl ketone ${ }^{9}$ and mesoporous crystalline material functionalised with mercaptopropyl $^{10}$ showed that these selectively extract $\mathrm{Hg}$ (II) from aqueous samples. Nevertheless, recovery and regeneration of the chelating agent is not practical. Considering this, a selective magnetic mercury scavenger would make the entire process much easier and faster as well as enhance the reusability of the chelating agent.

Indeed, functionally modified magnetic nanobeads have already been used for the extraction of different metal ions from 
aqueous solution based on cadmium, ${ }^{11,12}$ copper, ${ }^{11,13}$ lead, ${ }^{11,12}$ zinc, $^{13}$ mercury ${ }^{12,14,15}$ cobalt $^{16,17}$ and nickel ${ }^{16}$ under various conditions. However, concerning mercury limitations related to selectivity in the presence of other metals and reusability of the scavengers are being encountered. Iron oxide nanoparticles were primarily considered as an attractive solution for magnetic separation. Recently, Pang et $a l^{15}$ reported the synthesis of functionalised iron oxide nanoparticles which efficiently remove mercury(II) from water samples $\left(380 \mathrm{mg} \mathrm{Hg}^{2+}\right.$ extracted per mol adsorbent) but selectivity in combination with other metals or recyclability of this scavenger material was not studied. On the other hand, Khani et al. ${ }^{\mathbf{1 4}}$ have developed magnetite nanoparticles functionalised with triazene groups showing selectivity towards mercury in binary systems, which could be used in 2 cycles with an extraction capacity of $10.26 \mathrm{mg}$ $\mathrm{Hg}^{2+}$ per gram nanomaterial. Mandel et al. have reported that thiol-modified magnetic microparticles are capable of extracting mercury(II) preferentially over other metal ions. However, coadsorption of copper(II) and cadmium(II) was also observed in some cases. The release of adsorbed mercury(II) in order to recycle the scavenger was possible to an extent of about $30 \%$, and the estimated extraction capacity was around $74 \mathrm{mg} \mathrm{\textrm {Hg } ^ { 2 + }}$ per $\mathrm{g}$ microparticles. ${ }^{18}$ Magnetic Co/C nanoparticles, which exhibit excellent thermal and chemical stability as well as higher magnetization, recently appeared as a promising alternative $\mathbf{1 1 , 1 9}^{\mathbf{1 1 9}}$ for improving the extraction capacity and reusability of scavengers. Such nanoparticles provide an additional carbon surface that stabilizes the metal core and allows for functionalisation using established diazonium chemistry. ${ }^{20,21}$

Herein, we report the potential of $\mathrm{Co} / \mathrm{C}$ nanomagnets to be used as magnetic scavengers for mercury extraction from water. In addition, we studied the influence of amino functionalities on the nanoparticles to improve the extraction efficiency and selectivity, arriving at functional nanomagnets that show an extraction capacity for $\mathrm{Hg}^{2+}$ of up to $550 \mathrm{mg} \mathrm{g}^{-1} \mathrm{NP}$.

\section{Results and discussion}

Carbon-coated nanobeads have proved their effectiveness in a variety of applications such as supports for scavengers, reagents or catalysts. ${ }^{22-28}$ Relevant for this study, this type of nanoparticles was previously used for complexation/extraction of cadmium, copper, lead, ${ }^{\mathbf{1 1}}$ arsenic ${ }^{29}$ ions as well as noble metal ions based on gold ${ }^{19,30}$ and platinum..$^{30}$ However, no studies for the removal of mercury(II) from contaminated water were reported.

In order to remove $\mathrm{Hg}^{2+}$ ions from contaminated water, firstly pristine Co/C NPs $\mathbf{1}$, being commercially available ${ }^{20}$ were initially investigated as a possible scavenger. Two mercury solutions with different concentrations were prepared (15 and $30 \mathrm{mg} \mathrm{L}^{-1}$ ) and the progress of extraction was monitored by ICPOES during 10 minutes, aiming at practical decontamination times in real case scenarios and to study the adsorption kinetics and estimate the maximum extraction capacity of the nanobeads. From these results, using $5 \mathrm{mg}$ of NPs to decontaminate $5 \mathrm{~mL} \mathrm{HgCl}_{2}$ solution, it was concluded that approximately 13 $\mathrm{mg} \mathrm{Hg}^{2+}$ can be scavenged using $1 \mathrm{~g}$ of nanoparticles within 10 minutes, even at low initial mercury concentrations of $15 \mathrm{mg}$ $\mathrm{L}^{-1}$. However, also considerable leaching of Co ions from the nanoparticle core was observed. The adsorption of $\mathrm{Hg}^{2+}$ onto the carbon layer of the nanoparticles was confirmed by X-ray photoelectron spectroscopy (XPS) analysis (see ESI $\dagger$ ) and is in agreement with the results obtained for multi-walled carbon nanotubes (MWCNTs). ${ }^{31}$

Although the extraction of mercury(II) ions using unmodified $\mathrm{Co} / \mathrm{C}$ nanoparticles 1 proved to be efficient to some extent, there are three major limitations: (1) the occurring cobalt leaching leads to an undesired contamination that needs to be prevented. (2) The extraction capacity (13 $\mathrm{mg} \mathrm{Hg}^{2+}$ per $\mathrm{g} \mathrm{NPs}$ ) is relatively low requiring a high amount of nanoparticles to remove $\mathrm{Hg}^{2+}$ on large scale. (3) An efficient release of mercury from the particles, thus allowing their recycling was not possible under various conditions tried (aqua regia; heating at $150{ }^{\circ} \mathrm{C}$; aqua regia combined with high temperature).

Therefore, the surface of the nanomagnets was functionalised to improve the extraction capacity, also aiming to avoid cobalt leaching and ensuring recyclability. Non-magnetic amino-functionalised materials have been reported for their extraction capability towards mercury(II), and especially Masri and Friedman have demonstrated the high affinity of polyamine derivatives towards $\mathrm{Hg}^{2+}$ ions in aqueous solutions. ${ }^{32}$ Furthermore, amino-functionalised carbon nanotubes have been successfully applied for extracting mercury(II) from water samples. ${ }^{33}$ However, selectivity studies with these materials were either not performed or limited to binary systems. Taken these precedents as a lead, we aimed on developing high capacity amino-polymers, such as poly(ethyleneimine) (PEI) and poly(amidoamine) (PAMAM), supported on readily recyclable magnetic nanobeads for selective $\mathrm{Hg}^{2+}$ removal.

Thus, propargylated PAMAM dendrimer G2 having four terminal amino groups was connected in two different ways to the surface of the NPs (Scheme 1): benzylazide functionalised Co/C nanoparticles 2 (ref. 21 and 26) (0.1 mmol azide per $g$ nanomaterial) or a Wang type resin having azide end groups covalently attached to Co/C nanoparticles 3 (ref. 34) (2.4 mmol azide per $\mathrm{g}$ nanomaterial), were found to be suitable platforms to accommodate PAMAM dendrimers via ligation by a copper catalyzed azide/alkyne cycloaddition using conditions previously described by us. ${ }^{21,26}$ The reaction was conveniently followed by monitoring the characteristic azide peak at $2100 \mathrm{~cm}^{-1}$ with attenuated total reflection infrared spectroscopy (ATR-IR) spectroscopy (see Fig. S1, ESI $\dagger$ ), to give rise to $4(0.02 \mathrm{mmol}$ PAMAM per $\mathrm{g}$ nanomaterial) and 5 (0.57 mmol PAMAM per $\mathrm{g}$ nanomaterial), respectively (see Fig. S2, ESI $\dagger$ ). Higher magnetization values (Fig. S3, ESI $\dagger$ ) were observed for Co/C-PAMAM G2 4 (106 emu g ${ }^{-1}$ ) when compared to higher loaded Co/C-PSPAMAM G2 5 (50 $\mathrm{emu}^{-1}$ ), reflecting the different amounts of non-magnetic material attached to the nanobeads.

PEI-functionalised $\mathrm{Co} / \mathrm{C}$ nanobeads were prepared starting from 6 (ref. 20) ( $0.15 \mathrm{mmol}$ amine per $\mathrm{g}$ nanomaterial) following a procedure for the functionalization of carbon nanotubes described elsewhere (Scheme 2). ${ }^{35}$ Using 1000 equivalents of aziridine, high loadings of approximately $10 \mathrm{mmol}$ amine per $\mathrm{g}$ nanomaterial 7 were obtained by growing the PEI polymer on 


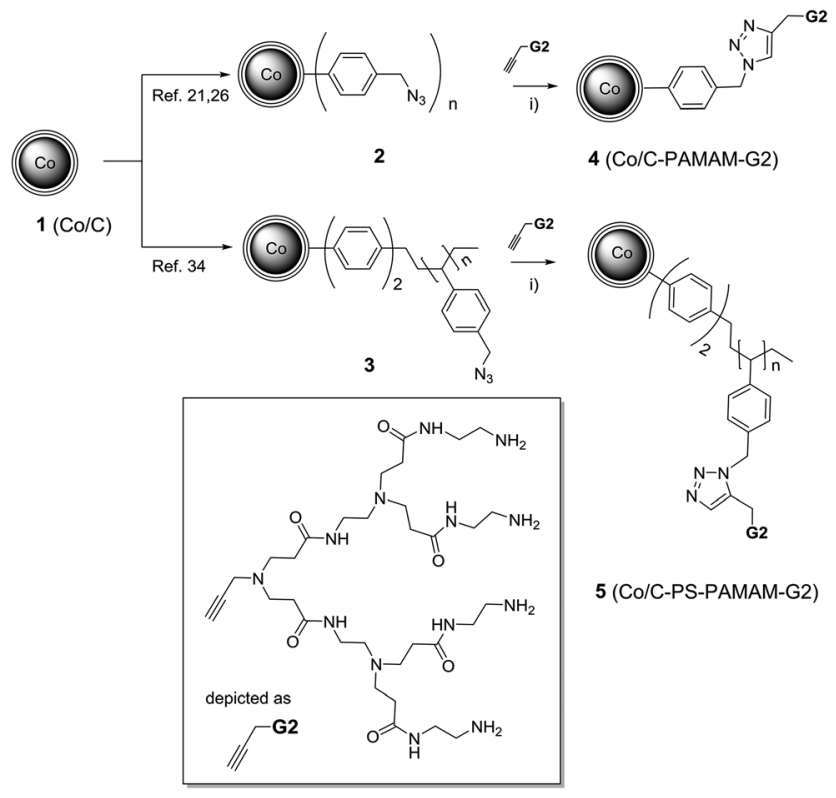

Scheme 1 Covalent immobilization of PAMAM dendron G2 on Co/C nanoparticles via click chemistry. Reagents and conditions: (i) $\mathrm{CuSO}_{4} \cdot 5 \mathrm{H}_{2} \mathrm{O}$ (10 mol\%), $\mathrm{Na}$ ascorbate (30 mol\%), THF- $\mathrm{H}_{2} \mathrm{O}$ (3: 1), 24 h, RT.

the nanoparticle surface. These nanoparticles form stable dispersions in water, ${ }^{36}$ thus avoiding agglomeration (see Fig. S6B and S7, ESI $\dagger$ ), which is a general problem for unmodified $\mathrm{Co} / \mathrm{C}$ nanoparticles. The saturation magnetization of this material was found to be still high (39 emu $\mathrm{g}^{-1}$, Fig. S6A, ESI $\dagger$ ), rivaling that of low-loading magnetite particles. ${ }^{37}$ Therefore, an easy and effective recovery by magnetic separation is still possible within seconds.

A comparison of the extraction efficiency of all nanobeads (Fig. 1) using $5 \mathrm{~mL}$ of an aqueous solution of $\mathrm{HgCl}_{2}\left(30 \mathrm{mg} \mathrm{L}^{-1}\right)$ and $5 \mathrm{mg}$ of nanomaterial during 10 minutes for benchmarking purposes showed that Co/C-PAMAM G2 4 was found to extract mercury (50\%) comparable to unmodified Co/C NPs, which is attributed to the low loadings obtained during the functionalization. Improved extraction capacity $(73 \%)$ was found for Co/C-PS-PAMAM G2 5, which can be ascribed to increased loadings of terminal amino groups made possible through the additional polystyrene layer on the surface of the NPs. ${ }^{24,25,29,34,38}$ For both materials no significant cobalt leaching was detected.

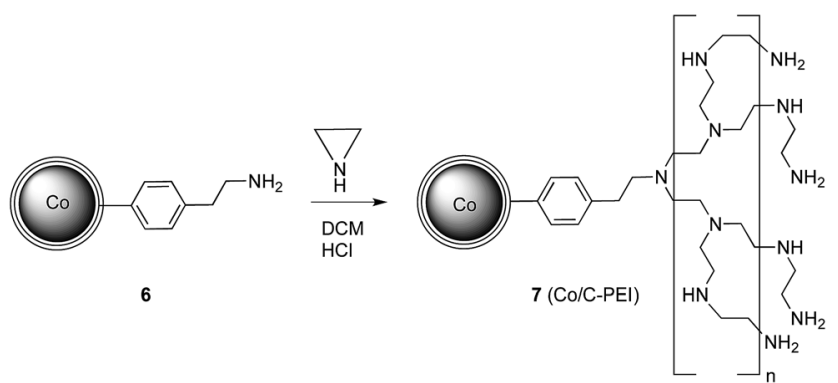

Scheme 2 Synthesis of poly(ethyleneimine) functionalised nanoparticles $7 .{ }^{36}$

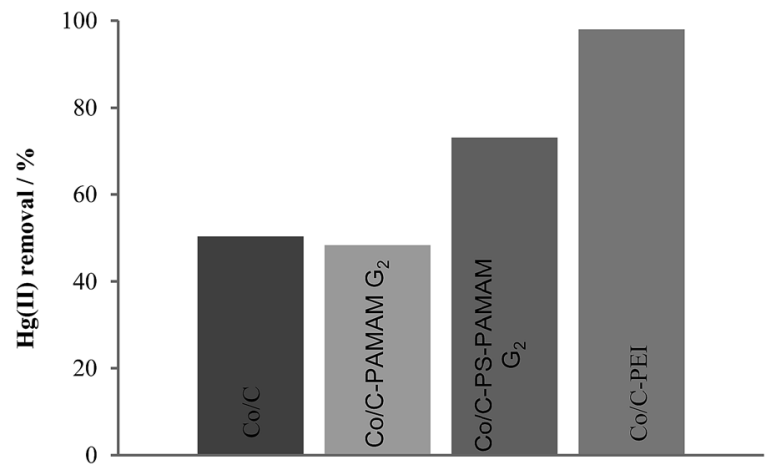

Fig. 1 Comparison of the extraction capacity from the different nanobeads (cf. Schemes 1 and 2). Reaction conditions: $5 \mathrm{mg}$ of NPs, $5 \mathrm{~mL}$ of $\mathrm{Hg}^{2+}$ solution $\left(30 \mathrm{mg} \mathrm{L}{ }^{-1}\right), 10$ min extraction time, solution $\mathrm{pH} 6.53$.

The $\mathrm{Hg}$ (II) removal efficiency was found to be even better ( $\geq 98 \%$, reaching the detection limit $\left[100 \mu \mathrm{g} \mathrm{L}{ }^{-1}\right]$ of the inductively coupled plasma optical emission spectrometry (ICP-OES) measurement) for $\mathrm{Co} / \mathrm{C}-\mathrm{PEI}$ 7, while still avoiding cobalt leaching from the nanoparticles into the solution. The maximum extraction capacity of Co/C-PEI 7 was subsequently estimated by extracting solutions of higher mercury concentration: the scavenging of $\mathrm{Hg}^{2+}$ from $5 \mathrm{~mL}$ of a $2.9 \mathrm{mM}$ solution with $5 \mathrm{mg}$ nanomaterial was still possible within 10 minutes to an extent of $95 \%$, corresponding to $550 \mathrm{mg} \mathrm{Hg}^{2+}$ extracted per $\mathrm{g}$ nanomaterial 7 , which compares favourably to the results obtained for $\mathrm{Co} / \mathrm{C} 1$ (15 $\mathrm{mg} \mathrm{\textrm {Hg } ^ { 2 + }}$ extracted per $\left.\mathrm{g} \mathrm{NPs}\right)$ and for previously reported magnetic mercury scavengers $(5.6-152 \mathrm{mg}$ $\mathrm{Hg}^{2+}$ extracted per $\mathrm{g}$ nanomaterial). ${ }^{4,14,39}$

Mercury(II) could also be efficiently removed from much more diluted solutions using 7: starting from $100 \mathrm{~mL}$ of an aqueous solution containing $1.87 \mathrm{mg} \mathrm{L}^{-1}$ mercury(II) chloride, just $3 \mathrm{mg} \mathrm{Co} / \mathrm{C}-\mathrm{PEI}$ nanoparticles 7 are sufficient to bring down again the mercury concentration to the detection limit (100 $\mu \mathrm{g} \mathrm{L}^{-1}$ ) of the ICP-OES analysis within 10 minutes (Fig. 2).

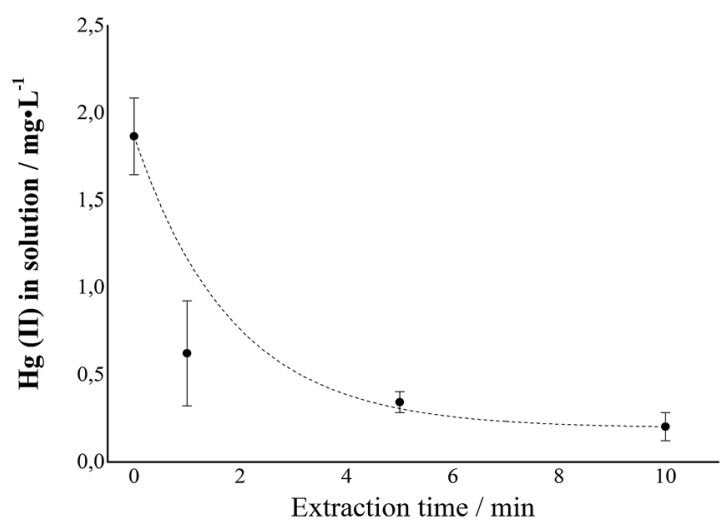

Fig. 2 Mercury(II) extraction over time. $100 \mathrm{~mL}$ of an aqueous solution (1.87 $\mathrm{mg} \mathrm{L}^{-1}$, solution $\mathrm{pH}$ 6.71), $3 \mathrm{mg} \mathrm{Co/C-PEI} \mathrm{nanoparticles} \mathrm{7.} \mathrm{The}$ dashed curve represents the exponential decay fit of the data set (decay constant: $1.8 \pm 1.2 \mathrm{~min}^{-1}$ ). After $10 \mathrm{~min}$, the detection limit $\left(100 \mu \mathrm{g} \mathrm{L}^{-1}\right)$ of the ICP-OES analysis was reached. 
To validate that the mercury(II) uptake occurs due to a complexation of the metal ions by the amino groups we tested the extraction capacity of the PEI-polymer itself. A commercially available PEG-resin with terminal amino groups 8 was functionalised with PEI in the same manner (Scheme 3) as for the Co/C-phenylethylamine particles 6 described above.

The so obtained PEI-resin 9 (10.9 mmol $\mathrm{N}$ per $\mathrm{g}$ resin) was used for extraction, applying identical conditions as in the previous experiments. A similar extraction capacity for the PEIfunctionalised resin 9 when compared to the Co/C-PEI nanobeads 7 was determined, while the PEG-amino resin 8 itself showed nearly no ability to extract mercury. These findings suggest that indeed the amino functionalities on the surface of the NPs are responsible for the removal of mercury(II), which is in agreement with literature reports for amino functionalised multi-wall carbon nanotubes ${ }^{33}$ or chitosan based absorbents ${ }^{39}$ as well as polyamine derivatives. ${ }^{32}$

It is known that PEI can also chelate metal ions such as $\mathrm{Ni}^{2+}, \mathrm{Cu}^{2+}, \mathrm{Zn}^{2+}, \mathrm{Cd}^{2+}$ and $\mathrm{Pb}^{2+} \cdot{ }^{40}$ However, to the best of our knowledge no selectivity studies using PEI for extracting mercury in the presence of other metal ions are reported. Testing the extraction of $\mathrm{Hg}^{2+}$ against other competitive metals when they are in solution at the same time, indeed we found that Co/C-PEI nanomagnets 7 show a high preference for mercury(II) (Fig. 3). Experiments were done with an extraction time of 10 minutes and 3 hours in a $\mathrm{pH}$ range of 5.6-6.2, representing the range that is obtained upon dissolving the metal salts in pure water. No significant differences were observed between these two time points indicating that under the conditions employed, after 10 minutes of extraction the equilibrium time for all metals tested has been reached. This result is supported by the selective extraction of mercury(II) shown also for the PEI-resin 9 (Fig. S9, ESI $\dagger$ ). Moreover, X-ray photoelectron spectroscopy (XPS) analysis on the NPs used to obtain the results in Fig. 3A confirmed the preferential uptake of mercury against the other metals (see $\mathrm{ESI} \dagger)$.

In addition, an experiment at basic $\mathrm{pH} 8.3$ was performed to evaluate the influence of the $\mathrm{pH}$ on the adsorption of the metals. Again, a preferential uptake of mercury was detected (68\%), however absorption of copper(II) (51\%) and lead(II) (17\%) (Fig. S10, ESI $\dagger$ ) occurred to a significant extent as well.

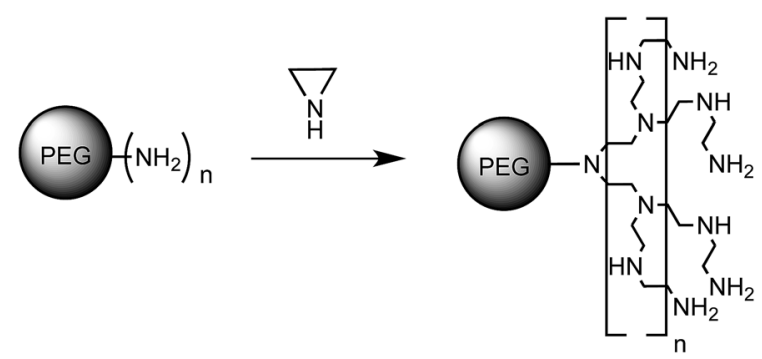

(NOVA PEG Amino Resin)

(NOVA PEG Amino Resin PEI)
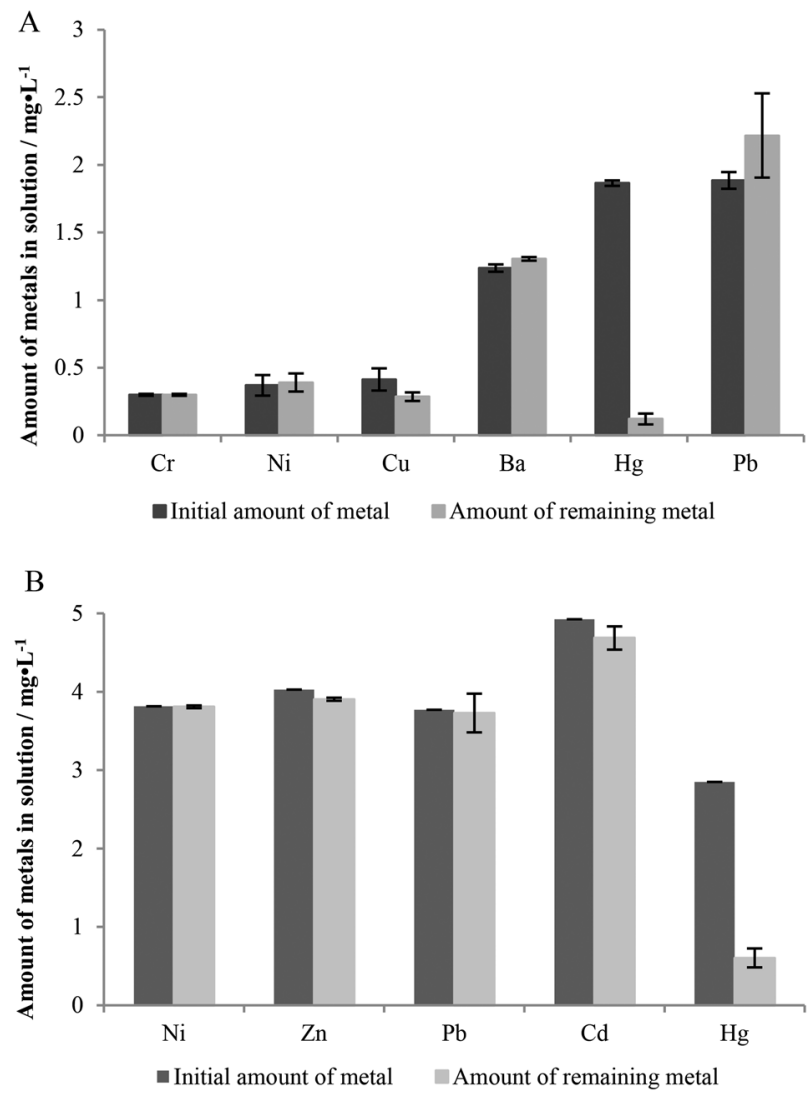

Fig. 3 Selective extraction of $\mathrm{Hg}^{2+}$ using CO/C-PEI 7 in the presence of competitive metal ions. (A) $3 \mathrm{mg}$ of NPs were used to decontaminate a $100 \mathrm{~mL}$ solution containing $\mathrm{Hg}^{2+}, \mathrm{Cu}^{2+}, \mathrm{Pb}^{2+}, \mathrm{Ni}^{2+}, \mathrm{Ba}^{2+}$ and $\mathrm{Cr}^{3+}$ in equimolar amounts $(10 \mu \mathrm{M})$, solution $\mathrm{pH}$ 5.59; (B) $3 \mathrm{mg}$ of NPs were used to decontaminate an aqueous $100 \mathrm{~mL}$ solution containing $\mathrm{Hg}, \mathrm{Pb}, \mathrm{Ni}, \mathrm{Zn}$ and $\mathrm{Cd}$, solution $\mathrm{pH} 6.16$.

Having developed a scavenger that combines the advantages of using a selective adsorbent with the magnetic properties of a solid support, we tested the performance of nanobeads 7 in tap water samples. For these experiments water from the facilities of the University of Regensburg was used and artificially contaminated with $\mathrm{Hg}^{2+}\left(2 \mathrm{mg} \mathrm{\textrm {L } ^ { - 1 }}\right)$. Especially, the water sample was analyzed with respect to the content of mercury, magnesium and iron before and after treatment with nanobeads 7 . The concentration of $\mathrm{Ca}^{2+}$ was also measured to be around $100 \mathrm{mg} \mathrm{L}^{-1}$, thus being present in large excess with respect to the extraction capacity of 7 used in this experiment. However, the values obtained from ICP measurements for calcium before and after extraction were somewhat erratic, which might be due to aging of the samples caused by carbon dioxide absorption. Despite the presence of those other ions that are naturally occurring in drinking water mercury was still efficiently removed (Table 1 , Sample 1).

As iron can occur in higher concentrations in water of different areas $^{\mathbf{4 1}}$ an additional experiment was performed in the presence of an excess of iron. Still $90 \% \mathrm{Hg}^{2+}$ is successfully extracted even if the content of iron is approximately 20 times higher than that of mercury (Table 1, Sample 2). 
Table 1 Extraction results in tap water

Metal ions before/after extraction $\left(\mathrm{mg} \mathrm{L}^{-1}\right)$

\begin{tabular}{lll}
\hline $\mathrm{Hg}^{c}$ & $\mathrm{Fe}^{c}$ & $\mathrm{Mg}^{c}$ \\
\hline $2.2 / 0.3$ & $\leq 0.1 / \leq 0.1$ & $19.1 / 19.1$ \\
$2.2 / 0.2$ & $35 / 32.5$ & -
\end{tabular}

Sample $1^{a}$ - Regensburg drinking water spiked with $\mathrm{Hg}^{2+}$

Sample $2^{b}$ - Regensburg drinking water spiked with $\mathrm{Fe}^{2+}$ and $\mathrm{Hg}^{2+}$
$2.2 / 0.3$
$2.2 / 0.2$

$35 / 32.5$

${ }^{a} \mathrm{Hg}^{2+}$ artificially added to the tap water samples (the source of mercury used was $\mathrm{HgCl}_{2}$ ). In addition, the sample contained approx. $100 \mathrm{mg} \mathrm{L}^{-1} \mathrm{Ca}^{2+}$ (see text). ${ }^{b} \mathrm{Fe}^{2+}$ and $\mathrm{Hg}^{2+}$ artificially added to the tap water samples (the source of iron used was $\mathrm{FeCl}_{2} \cdot 4 \mathrm{H}_{2} \mathrm{O}$ ). ${ }^{c}$ Values determined for tap water samples from the University of Regensburg. Extraction conditions: $3 \mathrm{mg}$ Co/C-PEI NPs 7 were used to decontaminate $100 \mathrm{~mL}$ aqueous solution (pH 6.71) within 10 minutes.

Having proven the feasibility of the nanomagnets for extracting mercury in real water samples, a simple recycling methodology of the magnetic scavenger had to be established. More specifically mercury has to be released after extraction in order to regenerate the nanomaterial and reuse it. Considering the fact that the amino groups in 7 are responsible for scavenging the mercury ions, a logical approach is the protonation of these groups using acidic conditions to reverse their complexation ability. For the release the following procedure was established: after the extraction time, the nanobeads were collected with a magnet and the aqueous decontaminated solution was completely decanted, followed by the addition of $20 \mathrm{~mL}$ of the corresponding acid. In the course of determining the conditions for the release of mercury different acids $(0.01 \mathrm{M})$ were tested. These experiments showed that strong acids like $\mathrm{H}_{2} \mathrm{SO}_{4}, \mathrm{HCl}$ and $\mathrm{HNO}_{3}$ are suitable for achieving high mercury release, while weak acids like acetic acid are less effective. Further optimizations were performed with $\mathrm{H}_{2} \mathrm{SO}_{4}$ solutions differing in molarity and thus in the $\mathrm{pH}$. The best conditions were found to be $0.5 \mathrm{M} \mathrm{H}_{2} \mathrm{SO}_{4}$, corresponding to a $\mathrm{pH}$ value of approximately 0.4. Noteworthy, ICP measurements revealed that no significant cobalt leaching from the core of the nanomaterial is detected during the release of mercury.

Thus, a multicycle extraction/recycling protocol was established (Fig. 4) for aqueous solutions (tap water) containing mercury. Briefly, the mercury(II) contaminated water containing the nanomagnets 7 is shaken for 10 minutes and then the NPs

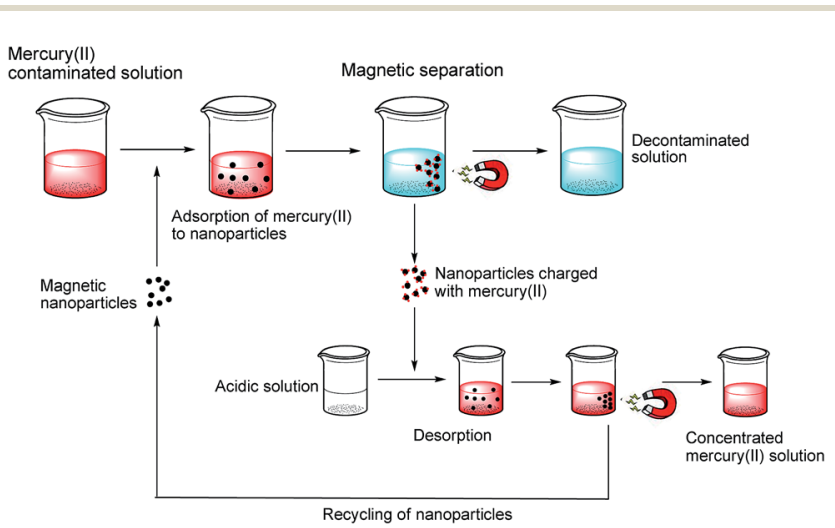

Fig. 4 Recycling protocol for the extraction of mercury in tap water samples. are recovered applying an external magnet. The decontaminated water is then decanted and the NPs 7 are subsequently treated with $\mathrm{H}_{2} \mathrm{SO}_{4}(20 \mathrm{~mL}, 0.5 \mathrm{M}, 20$ minutes) in order to release the mercury. Finally, a magnet is used once more to collect the NPs and decant the acidic solution which is followed by washing with a $0.5 \mathrm{M}$ potassium carbonate solution and water to regenerate the amino groups of 7 , and the nanobeads are used in the next cycle.

Following the scheme in Fig. 4, we demonstrate that in six consecutive cycles more than $90 \%$ of the mercury could be extracted from tap water samples $(6 \times 100 \mathrm{~mL}$ spiked with $2 \mathrm{mg}$ $\mathrm{L}^{-1} \mathrm{Hg}^{2+}$ each) within 10 minutes (Fig. 5). Even though the release step was not complete each time, the extraction capacity remained nearly unchanged during the six runs. In some cases the release is higher than $100 \%$ as mercury from a previous incomplete release step was apparently set free in the next cycle. In addition, TEM analysis (Fig. S11, ESI $\dagger$ ) proved that there are no significant changes or damage in the appearance of the nanoparticles after the recycling process. These results have encouraged us to study the applicability of these magnetic scavengers in a large-scale experiment aiming to prove their use in a realistic industrial application. For this purpose a $20 \mathrm{~L}$ reactor was used (see Fig. 6) and filled with normal Zurich drinking water artificially contaminated with $30 \mu \mathrm{g} \mathrm{L}^{-1} \mathrm{Hg}$ (II). An even lower

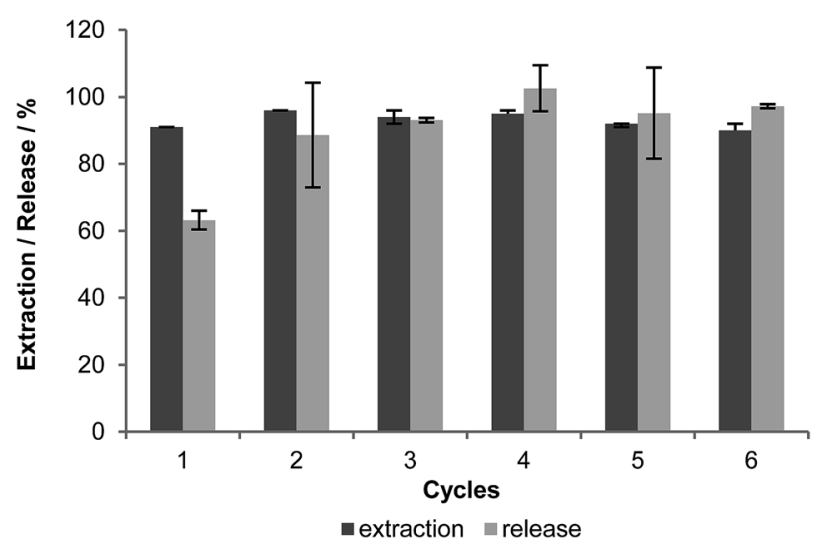

Fig. 5 Reusability of Co/C-PEI 7 in six consecutive runs (extraction and subsequent release). Extraction: Co/C-PEI $7(3 \mathrm{mg})$ were shaken in $100 \mathrm{~mL}$ of $2 \mathrm{mg} \mathrm{L}^{-1} \mathrm{Hg}($ II) containing aqueous sample (pH 6.7) for 10 min. Release: $20 \mathrm{~mL} 0.5 \mathrm{M} \mathrm{H}_{2} \mathrm{SO}_{4}$ within $20 \mathrm{~min}$. 


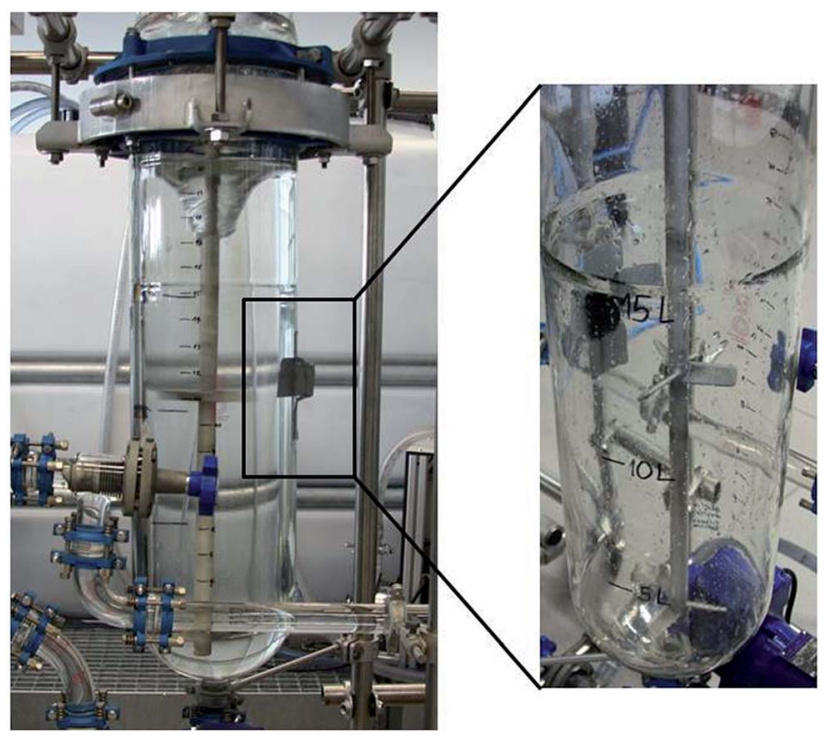

Fig. 6 Large-scale experiment was performed in a reactor containing $20 \mathrm{~L}$ of an aqueous mercury solution $\left(30 \mu \mathrm{g} \mathrm{L}^{-1}\right)$. The extraction was done at RT during one hour using 3 mg NPs 7 per liter, which were recovered by an external neodymium magnet (magnification, right picture).

concentration of particles than in the previous recycling experiments was employed $\left(3 \mathrm{mg} \mathrm{L}^{-1}\right)$, gratifyingly, after one hour reaction time the water was detoxified from mercury to $93 \%$, leaving behind a mercury content of $2 \mu \mathrm{g} \mathrm{L} \mathrm{L}^{-1}$ as determined by atomic fluorescence spectroscopy (AFS), which is within the limit for drinking water according to World Health Organization. ${ }^{41}$ Thus, the simple and efficient scavenger developed here has proved its potential to decontaminate water samples from mercury(II) poisoning, which also might be applicable in the open environment due to the facile recovery of the magnetic support.

\section{Experimental}

Full information on the materials and the equipment used as well as the detailed syntheses and characterization is provided in the ESI. $\dagger$ The extraction capacity of the nanomaterials described is expressed as mg mercury per $g$ nanomaterial.

\section{Nomenclature of nanobeads}

The nomenclature of the nanoparticles is as follows: $\mathrm{Co} / \mathrm{C}$ for magnetic nanoparticles with cobalt core and carbon shell. Co/ $\mathrm{C}-\mathrm{R}$ for functionalised $\mathrm{Co} / \mathrm{C}$ NPs where R indicates the functional groups on the graphene-like layers: PAMAM G2 for the dendrimeric poly(amidoamine) coating of the second generation and PEI for the poly(ethyleneimine) coating. Co/C-PSPAMAM G2 for polystyrene coated cobalt nanoparticles with an additional dendrimeric functionalization.

\section{Adsorption of mercury from aqueous solutions}

A given amount of the magnetic nanoparticles was added to an aqueous mercury solution with a defined concentration of the heavy metal. The experiment was carried out at room temperature and the $\mathrm{pH}$ of the solutions specified at the results section. The metal salts used are $\mathrm{HgCl}_{2}, \mathrm{BaCl}_{2} \cdot 2 \mathrm{H}_{2} \mathrm{O}, \mathrm{CuCl}_{2}$, $\mathrm{CrCl}_{3} \cdot 6 \mathrm{H}_{2} \mathrm{O}, \mathrm{PbCl}_{2}, \mathrm{Ni}\left(\mathrm{C}_{5} \mathrm{H}_{7} \mathrm{O}_{2}\right)_{2}, \mathrm{Zn}\left(\mathrm{ClO}_{4}\right) 2 \cdot 6 \mathrm{H}_{2} \mathrm{O}, \mathrm{FeCl}_{2} \cdot 4 \mathrm{H}_{2} \mathrm{O}$ and $\mathrm{CdCl}_{2} \cdot \mathrm{H}_{2} \mathrm{O}$.

First, the nanobeads in solution were dispersed for one minute in the ultrasonic bath and then the dispersion was agitated in a mechanical shaker for the rest of the extraction time. Afterwards the nanobeads were collected with the help of a magnet and the remaining solution was decanted. The remaining mercury in solution was determined by inductively coupled plasma optical emission spectrometry analysis (ICPOES, detection limit: $0.1 \mathrm{mg} \mathrm{L}^{-1}$ ). For the large scale experiment the remaining solution was analysed by atomic fluorescence spectroscopy (AFS) with a detection limit of $1 \mu \mathrm{g} \mathrm{L} \mathrm{L}^{-1}$.

\section{Desorption of mercury and re-usability of the nanomagnets}

The release of mercury from the magnetic support was performed in acidic medium. The amount of nanoparticles used for the extraction $(3 \mathrm{mg}$ ) was dispersed in $20 \mathrm{~mL}$ of the specified acid and then sonicated for $3 \mathrm{~min}$ followed by $5 \mathrm{~min}$ of mechanical shaking. The nanobeads were collected using an external magnet, washed with a $0.5 \mathrm{M}$ potassium carbonate solution and water and re-used for the next adsorption experiment. This procedure was repeated 6 times to study the materials' recyclability and reused for the next cycle.

To determine the amount of mercury desorbed, the acid solution used above was diluted with aqua regia $32 \%(\mathrm{v} / \mathrm{v})$, filtered and analyzed by ICP-OES.

\section{Conclusions}

Unfunctionalised carbon-coated nanobeads 1 proved to have potential for mercury removal from water, however, with some major limitations. A significant improvement was achieved with PEI-functionalised nanomagnets 7 , which showed a very high capacity for extracting toxic $\mathrm{Hg}^{2+}$ in a multimetal environment from drinking water samples at relevant concentrations. The extraction occurs through the complexation of $\mathrm{Hg}^{2+}$ ions by the amino groups of the functionalised nanoparticles 7 .

The recyclability of the nanoparticles was ensured for at least 6 consecutive cycles with no loss of extraction capacity. The particles 7 showed as well the ability of extracting from a $20 \mathrm{~L}$ reactor, which proved the potential of 7 for the detoxification of drinking water in realistic applications.

In summary, we have developed a simple and efficient scavenger to decontaminate water samples from mercury(II) poisoning, which might also be applicable in the open environment due to the facile recovery of the magnetic support.

\section{Acknowledgements}

Financial support by the EU-ITN network Mag(net)icFun (PITNGA-2012-290248) and the Hanns-Seidel-Stiftung (fellowship for C. M. E.) is gratefully acknowledged. We are grateful to Dr Rainer Müller for measuring the TGA, Dr Quirin Kainz for providing starting material for the synthesis of magnetic Co/C-PEI. 


\section{Notes and references}

1 A. Farrukh, A. Akram, A. Ghaffar, S. Hanif, A. Hamid, H. Duran and B. Yameen, ACS Appl. Mater. Interfaces, 2013, 5, 3784 .

2 H. Parham, B. Zargar and R. Shiralipour, J. Hazard. Mater., 2012, 205-206, 94.

3 M. H. Baki and F. Shemirani, Anal. Methods, 2013, 5, 3255.

4 J. Zhao, B. Zhu, H. Yu, L. Yan, Q. Wei and B. Du, J. Colloid Interface Sci., 2013, 389, 46.

5 Y. Zhai, S. Duan, Q. He, X. Yang and Q. Han, Microchim. Acta, 2010, 169, 353.

6 P. I. Girginova, A. L. Daniel-da-Silva, C. B. Lopes, P. Figueira, M. Otero, V. S. Amaral, E. Pereira and T. Trindade, J. Colloid Interface Sci., 2010, 345, 234.

7 (a) C. C. Freyhardt, M. Tsapatsis, R. F. Lobo, K. J. Balkus and M. E. Davis, Nature, 1996, 381, 295; (b) K. Inoue, K. Yoshizuka and K. Ohto, Anal. Chim. Acta, 1999, 388, 209; (c) A. K. Kushwaha, N. Gupta and M. C. Chattopadhyaya, J. Chem. Pharm. Res., 2011, 807.

8 M. A. Shannon, P. W. Bohn, M. Elimelech, J. G. Georgiadis, B. J. Mariñas and A. M. Mayes, Nature, 2008, 452, 301.

9 D. Astruc, C. Ornelas and J. Ruiz, Acc. Chem. Res., 2008, 41, 841.

10 J. N. H. Reek, S. Arévalo, R. van Heerbeek, P. C. J. Kamer and P. W. N. M. van Leeuwen, in Advances in Catalysis, ed. C. Bruce, Gates and Helmut Knözinger, Academic Press, 2006, vol. 49, pp. 71-151.

11 F. M. Koehler, M. Rossier, M. Waelle, E. K. Athanassiou, L. K. Limbach, R. N. Grass, D. Günther and W. J. Stark, Chem. Commun., 2009, 4862.

12 J.-f. Liu, Z.-s. Zhao and G.-b. Jiang, Environ. Sci. Technol., 2008, 42, 6949.

13 Y. Zhai, Q. He, Q. Han and S. Duan, Microchim. Acta, 2012, 178, 405.

14 M. K. Rofouei, A. Rezaei, M. Masteri-Farahani and H. Khani, Anal. Methods, 2012, 4, 959.

15 X. Shen, Q. Wang, W. Chen and Y. Pang, Appl. Surf. Sci., 2014, 317, 1028.

16 M. Faraji, Y. Yamini, A. Saleh, M. Rezaee, M. Ghambarian and R. Hassani, Anal. Chim. Acta, 2010, 659, 172.

17 Y. Wang, X. Luo, J. Tang, X. Hu, Q. Xu and C. Yang, Anal. Chim. Acta, 2012, 713, 92.

18 K. Mandel, F. Hutter, C. Gellermann and G. Sextl, ACS Appl. Mater. Interfaces, 2012, 4, 5633.

19 M. Rossier, F. M. Koehler, E. K. Athanassiou, R. N. Grass, B. Aeschlimann, D. Günther and W. J. Stark, J. Mater. Chem., 2009, 19, 8239.
20 R. N. Grass, E. K. Athanassiou and W. J. Stark, Angew. Chem., Int. Ed., 2007, 46, 4909.

21 Q. M. Kainz, A. Schätz, A. Zöpfl, W. J. Stark and O. Reiser, Chem. Mater., 2011, 23, 3606.

22 Q. M. Kainz, R. Linhardt, R. N. Grass, G. Vilé, J. PérezRamírez, W. J. Stark and O. Reiser, Adv. Funct. Mater., 2014, 24, 2020.

23 Q. M. Kainz, R. Linhardt, P. K. Maity, P. R. Hanson and O. Reiser, ChemSusChem, 2013, 6, 721.

24 Q. M. Kainz and O. Reiser, Acc. Chem. Res., 2014, 47, 667.

25 Q. M. Kainz, M. Zeltner, M. Rossier, W. J. Stark and O. Reiser, Chem.-Eur. J., 2013, 19, 10038.

26 A. Schätz, R. N. Grass, W. J. Stark and O. Reiser, Chem.-Eur. J., 2008, 14, 8262.

27 M. Keller, V. Collière, O. Reiser, A.-M. Caminade, J.-P. Majoral and A. Ouali, Angew. Chem., Int. Ed., 2013, 52, 3626.

28 S. Wittmann, A. Schätz, R. N. Grass, W. J. Stark and O. Reiser, Angew. Chem., Int. Ed., 2010, 49, 1867.

29 M. Rossier, A. Schaetz, E. K. Athanassiou, R. N. Grass and W. J. Stark, Chem. Eng. J., 2011, 175, 244.

30 M. Rossier, F. M. Koehler, E. K. Athanassiou, R. N. Grass, M. Waelle, K. Birbaum, D. Günther and W. J. Stark, Ind. Eng. Chem. Res., 2010, 49, 9355.

31 B. Tawabini, S. Al-Khaldi, M. Atieh and M. Khaled, Water Sci. Technol., 2010, 61, 591.

32 M. S. Masri and M. Friedman, Environ. Sci. Technol., 1972, 6, 745.

33 M. Soleimani, M. Ghahraman Afshar and A. Sedghi, ISRN Nanotechnol., 2013, 674289, DOI: 10.1155/2013/674289.

34 M. Keller, A. Perrier, R. Linhardt, L. Travers, S. Wittmann, A.-M. Caminade, J.-P. Majoral, O. Reiser and A. Ouali, Adv. Synth. Catal., 2013, 355, 1748.

35 Y. Liu, D.-C. Wu, W.-D. Zhang, X. Jiang, C.-B. He, T. S. Chung, S. H. Goh and K. W. Leong, Angew. Chem., Int. Ed., 2005, 44, 4782.

36 Q. M. Kainz, S. Fernandes, C. M. Eichenseer, F. Besostri, H. Körner, R. Müller and O. Reiser, Faraday Discuss., 2015, 175, 27.

37 A. K. Tucker-Schwartz and R. L. Garrell, Chem.-Eur. J., 2010, 16, 12718.

38 A. Schaetz, M. Zeltner, T. D. Michl, M. Rossier, R. Fuhrer and W. J. Stark, Chem.-Eur. J., 2011, 17, 10566.

39 G. Z. Kyzas and E. A. Deliyanni, Molecules, 2013, 18, 6193.

40 C. Bazzicalupi, A. Bianchi, C. Giorgi, P. Gratteri, P. Mariani and B. Valtancoli, Inorg. Chem., 2013, 52, 2125.

41 Iron in drinking water, Guidelines for drinking-water quality, World Health Organization, Geneva, 2nd edn, 1996. 